\title{
Effect of double-polarization data in fits to single-pion photoproduction
}

\author{
Richard A. Arndt, Igor I. Strakovsky, and Ron L. Workman \\ Center for Nuclear Studies, Department of Physics, \\ The George Washington University, Washington DC 20052
}

\begin{abstract}
We investigate the influence of new Jefferson Lab polarization-transfer, $C_{x^{\prime}}$ and $C_{z^{\prime}}$, and recoil polarization, $P_{y}$, measurements in multipole fits to the single-pion photoproduction database. Results are compared to those found with the addition of a double-polarization quantity associated with the Gerasimov-Drell-Hearn sum rule.

PACS numbers: PACS numbers: 25.20.Lj, 13.60.Le, 11.40.-q, 11.80.Cr
\end{abstract}

The single-pion photoproduction process has been studied extensively, both experimentally and theoretically, for several decades. Over the resonance region, there now exists a database of approximately twenty thousand angular and total cross section measurements. This set consists mainly of unpolarized cross sections, many of these having been obtained without the benefit of tagged photons.

Experimental programs now underway have been adding very precise cross sections, many involving tagged photons, polarization, and a wide angular acceptance. Coverage is not yet complete. As a result, our knowledge of the underlying resonance contributions is based on a mix of data, old and new, some of which are certainly flawed. Fits to the full data set tend to favor the newer measurements [1]. However, experience has shown that sensitive quantities can be biased by older results, particularly if they cover a gap in the newer measurements, and are presented with comparable uncertainties [2].

Polarization quantities have been particularly influential in constraining multipole analyses. For this process, there are 8 independent quantities, each providing a new constraint on the 4 helicity amplitudes. In the $\Delta(1232)$ region, a single resonance is dominant, and in this case (only) each new quantity can be translated into a constraint on a single $\left(M_{1+}^{3 / 2}\right)$ multipole and its interference with other smaller multipoles. Here, and also at higher energies, the beam-polarization $(\Sigma)$ has been useful in revealing problems [3] with the existing SAID [1] and MAID 渵 fits.

Until recently, very few double-polarization measurements existed, and the available data had large uncertainties, weakening their influence in unweighted fits. As a by-product of the ongoing GDH measurements, angular distributions of the difference of helicity $1 / 2$ and $3 / 2$ cross sections, related to the beam-target polarization quantity $(E)$ [5], have been measured and fitted [6]. While SAID and MAID gave qualitatively correct predictions for this quantity, deviations starting at $700 \mathrm{MeV}$ were linked to the $N(1520)$ photo-decay amplitudes $[6]$.

A double-polarization experiment [7], giving the new beam-recoil quantities $C_{x^{\prime}}$ and $C_{z^{\prime}}$, has been completed by the Hall A Collaboration at Jefferson Lab. These measurements, which extend from $0.83 \mathrm{GeV}$ to $4.03 \mathrm{GeV}$, are given with high precision, and cover a region dominated by older data. While SAID and MAID predict the lowest energy results, at energies of $1 \mathrm{GeV}$ and beyond even qualitative agreement is lost [8]. In addition, just beyond the energy range of SAID, the recoil polarization $\left(P_{y}\right)$, also measured in Ref. [7], appears to have a rapid angular variation not seen below $2 \mathrm{GeV}$.

We have attempted to fit these new values of $P_{y}, C_{x^{\prime}}$, and $C_{z^{\prime}}$ in order to determine their effect on our multipole solution. In Figs. 1 and 2, we compare our results (SM02) prior to the measurement of Ref. [7], (SG02) including the new $P_{y}, C_{x^{\prime}}$, and $C_{z^{\prime}}$ points, and (SC02) a forced fit. At $815 \mathrm{MeV}$ and $1215 \mathrm{MeV}$, comparisons with MAID are also presented. The forced fit has weighted the data of Ref. [7] by a factor of 4 . By weighting data, we magnify changes in the multipole amplitudes, and more clearly see where data conflicts occur. In the case of the E2/M1 ratio, for example, an early forced fit [9] to $\Sigma$ data resulted in a value of $-2.9 \%$, when the predominant value was $-1.5 \%$.

In Figs. 1 and 2, it is clear that both SAID and MAID, at the lower energies where they can be compared, have a shape consistent with the $C_{x^{\prime}, z^{\prime}}$ data. The deviations at $1620 \mathrm{MeV}$ and $1900 \mathrm{MeV}$ are much larger. However, except for a few points, the fit including data from Ref. [7] is not appreciably different from the "forced" fit, and appears to adequately describe these new results. In Fig. 3, we show that forcing a fit to this new set has not degraded our fit to the GDH measurements. Other double-polarization observables deviate considerably, according to the weighting of these polarization-transfer data. These revised fits have been carried out using the 
parametrization described in Ref. [1]. No increase in the angular momentum limit for parametrized waves was found to be necessary.

In Fig. 目, we display the above fits for $P_{y}$. As more weight is given to the measurements of Ref. [7], an interesting feature emerges in the angular distribution at $1900 \mathrm{MeV}$. A sharp structure at about $60^{\circ}$ is seen in the forced fit. This is correlated with the improved fit seen in Fig. 2(d), for $C_{z^{\prime}}$, and Fig. 3(b), for the targetpolarization quantity $T$. This feature of the single-spin asymmetries is not new, however, and was present in the old analysis of Barbour, Crawford, and Parsons 10.

The changes displayed in Figs. 11 through 10 cannot be attributed to a single resonance or partial wave, as was argued for the GDH observable in Ref. [6]. A number of multipoles show moderate changes [14], and only some qualitative observations are possible. For example, a change in the $E_{0+}^{3 / 2}$ multipole can effect the overall shift seen in Fig. 1. 1 (d), and changes in either the $M_{1+}^{1 / 2}$ or $M_{1+}^{3 / 2}$ multipoles produce a peaking structure such as is seen in Figs. 3(b) and (d). However, a more complicated change in several amplitudes has given the full result. Some of the most prominent changes are displayed in Fig. 5 .

In summary, we have found it possible to fit recent double-polarization measurements which initially showed significant deviations from both SAID and MAID. Adding these precise new data has actually improved our fit to some other quantities, most obviously the target-polarization asymmetry, and has been accomplished without a substantial degradation of our fit to other observables. The resulting shifts in our multipoles indicate that the region above $1 \mathrm{GeV}$ is still underconstrained. Further shifts should be expected as the present generation of photon facilities contributes data to this region. In this regard, the proposed polarized-target/beam program for Hall B of JLab would be extremely useful 15.

\section{Acknowledgments}

We thank R. Gilman for helpful discussions. This work was supported in part by the U. S. Department of Energy Grant DE-FG02-99ER41110. The authors gratefully acknowledge a contract from Jefferson Lab under which this work was done. Jefferson Lab is operated by the Southeastern Universities Research Association under the U. S. Department of Energy Contract DE-AC0584ER40150.
[1] R. A. Arndt, W. J. Briscoe, I. I. Strakovsky, and R. L. Workman, Phys. Rev. C 66, 055213 (2002).

[2] R. M. Davidson, N. C. Mukhopadhyay, M. S. Pierce, R. A. Arndt, I. I. Strakovsky, and R. L. Workman, Phys. Rev. C 59, 1059 (1999).

[3] O. Bartalini et al., Phys. Lett. B544, 112 (2002); J. Ajaka et al., Phys. Lett. B475, 372 (2000).

[4] Mainz fits are available at the MAID website http://www.kph.uni-mainz.de/MAID/. See also S. S. Kamalov, S. N. Yang, D. Drechsel, O. Hanstein, and L. Tiator, Phys. Rev. C 64, 032201 (2001). The Unitary Isobar Model was developed at Mainz, D. Drechsel, O. Hanstein, S. S. Kamalov, and L. Tiator, Nucl. Phys. A645, 145 (1999). MAID refers to the Nov. 2001 version of the MAID solution from S. Kamalov.

[5] I. S. Barker, A. Donnachie, and J. K. Storrow, Nucl. Phys. B95, 347 (1975).

[6] J. Ahrens et al., Phys. Rev. Lett. 88, 232002 (2002).

[7] K. Wijesooriya et al., Phys. Rev. C 66, 034614 (2002).

[8] Comparison of SAID and MAID is limited by the MAID energy range, which is expected increase in future versions (S. Kamalov, private communication).
[9] R. L. Workman, R. A. Arndt, and Z. Li, Phys. Rev. C 46, 1546 (1992).

[10] I. M. Barbour, R. L. Crawford, and N. H. Parsons, Nucl. Phys. B141, 253 (1978); P. J. Bussey et al., Nucl. Phys. B154, 492 (1979).

[11] P. J. Bussey et al., Nucl. Phys. B159, 383 (1979); P. S. L. Booth, DNPL Annual Report, 1977.

[12] R. O. Avakyan et al., Yad. Fiz. 53, 717 (1991) [Sov. J. Nucl. Phys. 53, 448 (1991)].

[13] The full database and numerous PWAs can be accessed via a ssh/telnet call to the SAID facility gwdac.phys.gwu.edu, with userid: said (no password), or a link to the website http://gwdac.phys.gwu.edu.

[14] While it is possible to improve the fit by changing a single multipole, as was found for the GDH data, this approach tends to degrade the fit to unpolarized cross section, as is evident in Fig. 2 of Ref. [6]. This was also mentioned by, R. Workman, R. A. Arndt, and I. I. Strakovsky, Phys. Rev. C 62, 048201 (2000).

[15] R. A. Arndt et al., JLab Letter-of-Intent, LoI-02-101, Newport News, VA, USA, 2002. 

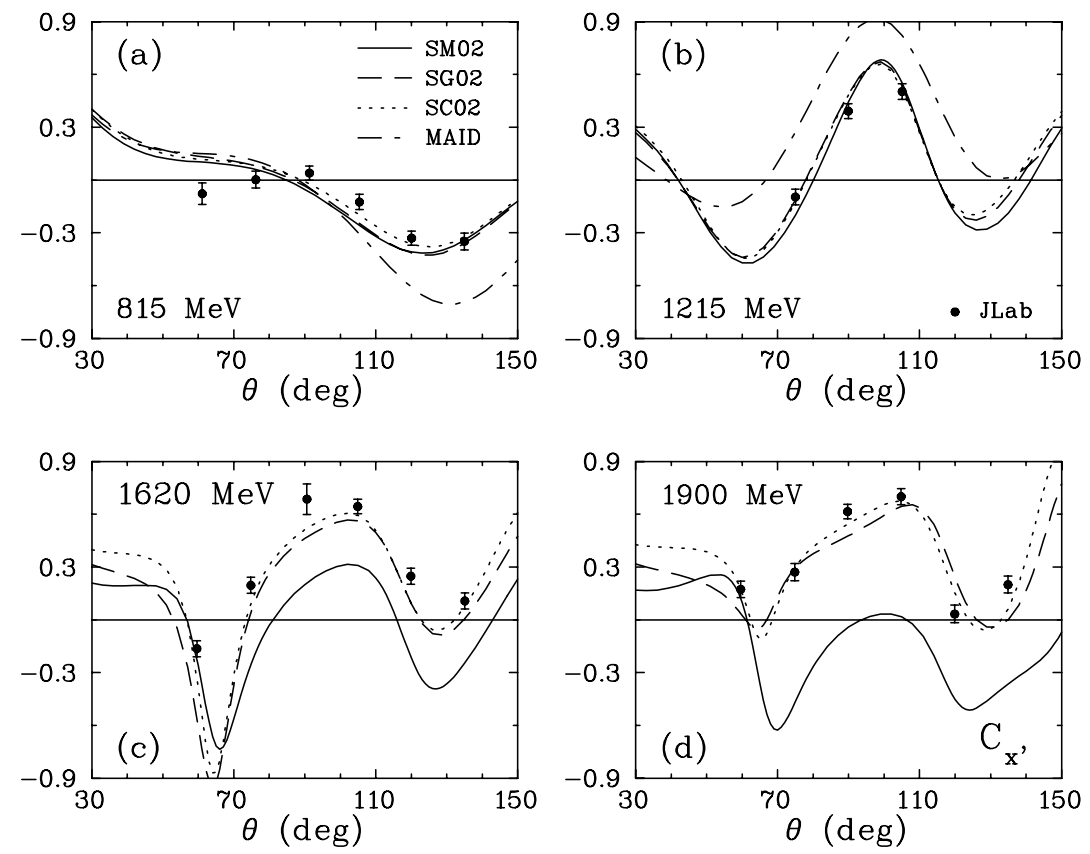

FIG. 1: Polarization transfer $C_{x^{\prime}}$ in neutral pion photoproduction at medium angles in the fixed lab frame: (a) $\mathrm{E}_{\gamma}=815 \mathrm{MeV}$, (b) $1215 \mathrm{MeV}$, (c) $1620 \mathrm{MeV}$, and (d) $1900 \mathrm{MeV}$. Solid (dashed-dot) line gives the SM02 值 (MAID 四) solution. Dashed (dotted) line shows the standard SG02 (forced SC02) solution including the data of Ref. [7]. Plots (a) and (b) have centered angular points within a $18 \mathrm{MeV}$ bin; plots (c) and (d) within a $45 \mathrm{MeV}$ bin.
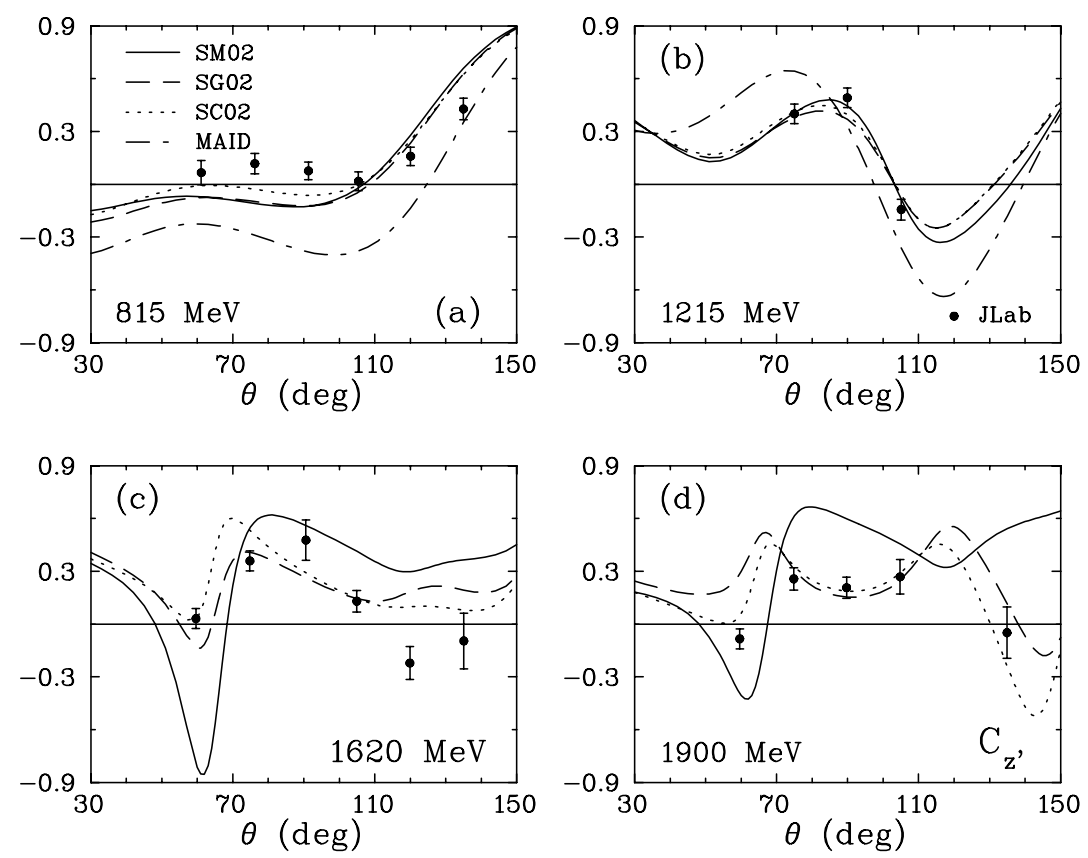

FIG. 2: Polarization transfer $C_{z^{\prime}}$ in neutral pion photoproduction at medium angles in the fixed lab frame. The notation is the same as for Fig. 1. 

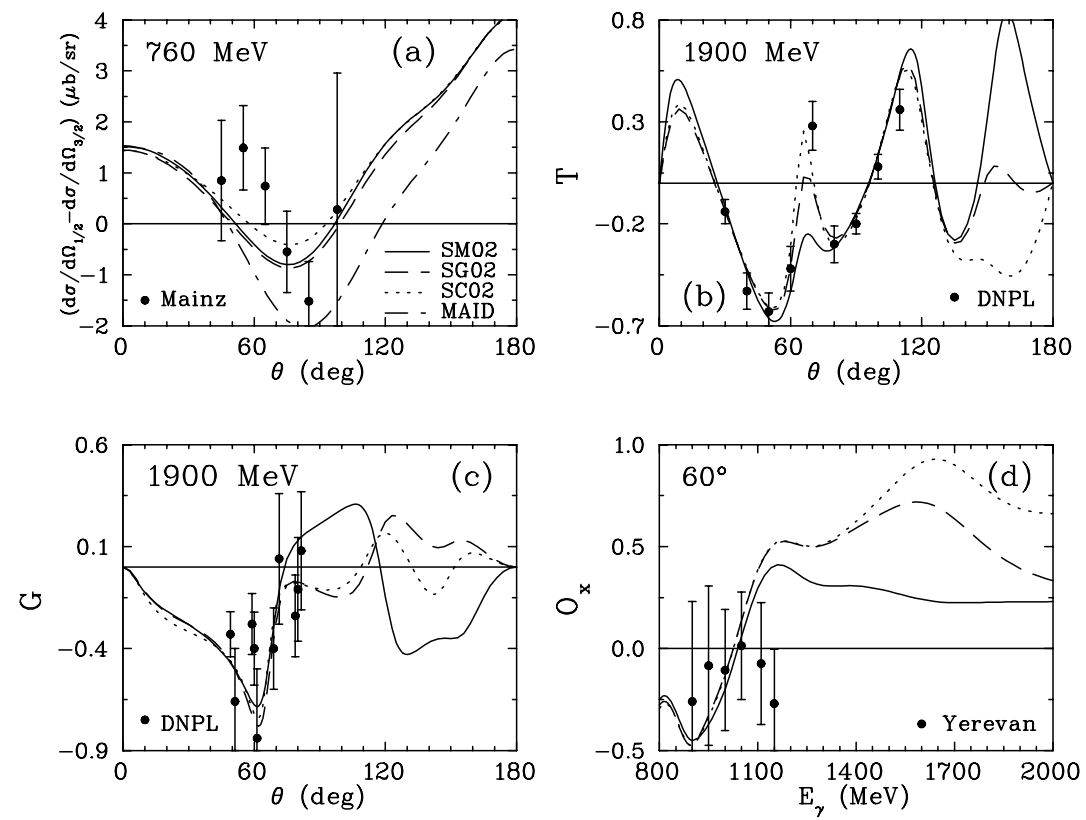

FIG. 3: Polarized measurements in neutral pion photoproduction: (a) $d \sigma / d \Omega_{1 / 2}-d \sigma / d \Omega_{3 / 2}$, (b) T, (c) G, and (d) $O_{x}$. The notation for curves is the same as for Fig. 11. Data for (a) are from Mainz [6], data for (b) and (c) are from DNPL 10, 11], and data for (d) are from Yerevan [12].
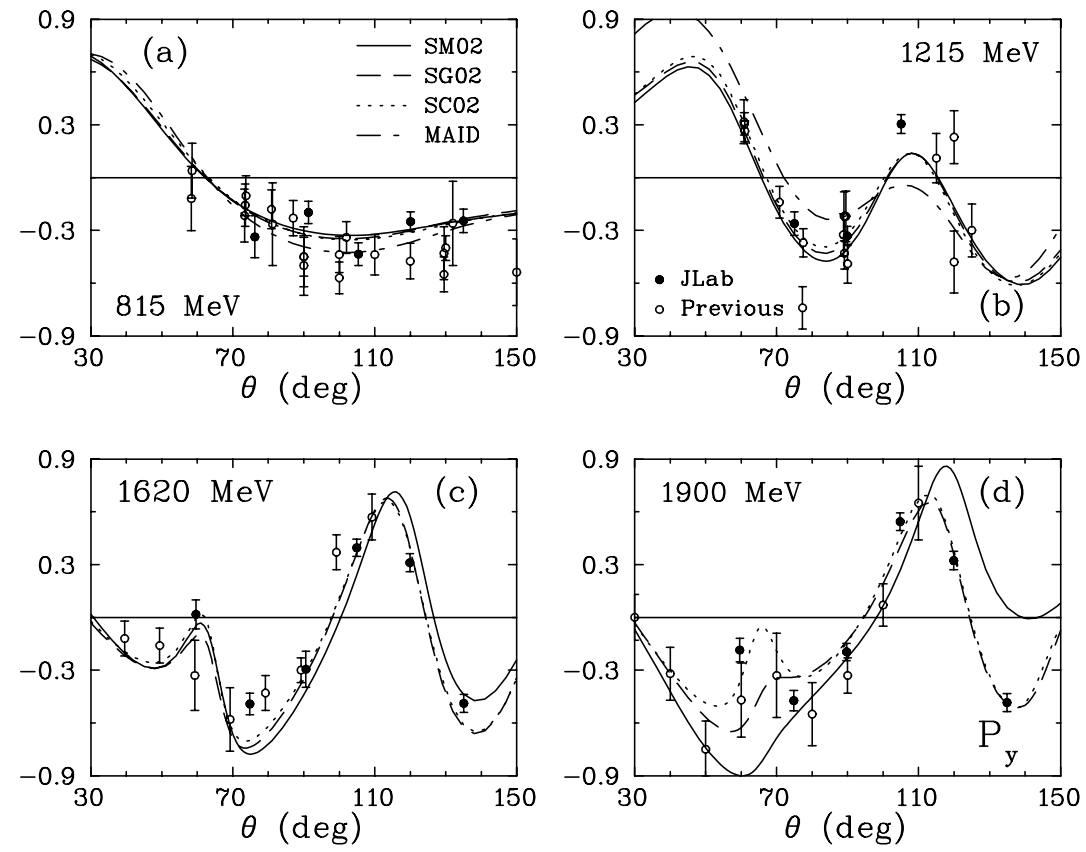

FIG. 4: Induced polarization $P_{y}$ in neutral pion photoproduction at medium angles. The notation for curves is the same as for Fig. 1. Previous Bonn, DNPL, Frascati, Kharkov, SLAC, Tokyo, and Yerevan measurements are available in the SAID database 13. (a) $815 \mathrm{MeV}$ plot covers 799 to $825 \mathrm{MeV}$ range, (b) $1205 \mathrm{MeV}$ plot covers 1192 to $1225 \mathrm{MeV}$ range, (c) $1620 \mathrm{MeV}$ plot covers 1600 to $1639 \mathrm{MeV}$ range, and (d) $1900 \mathrm{MeV}$ plot covers 1876 to $1921 \mathrm{MeV}$ range. 

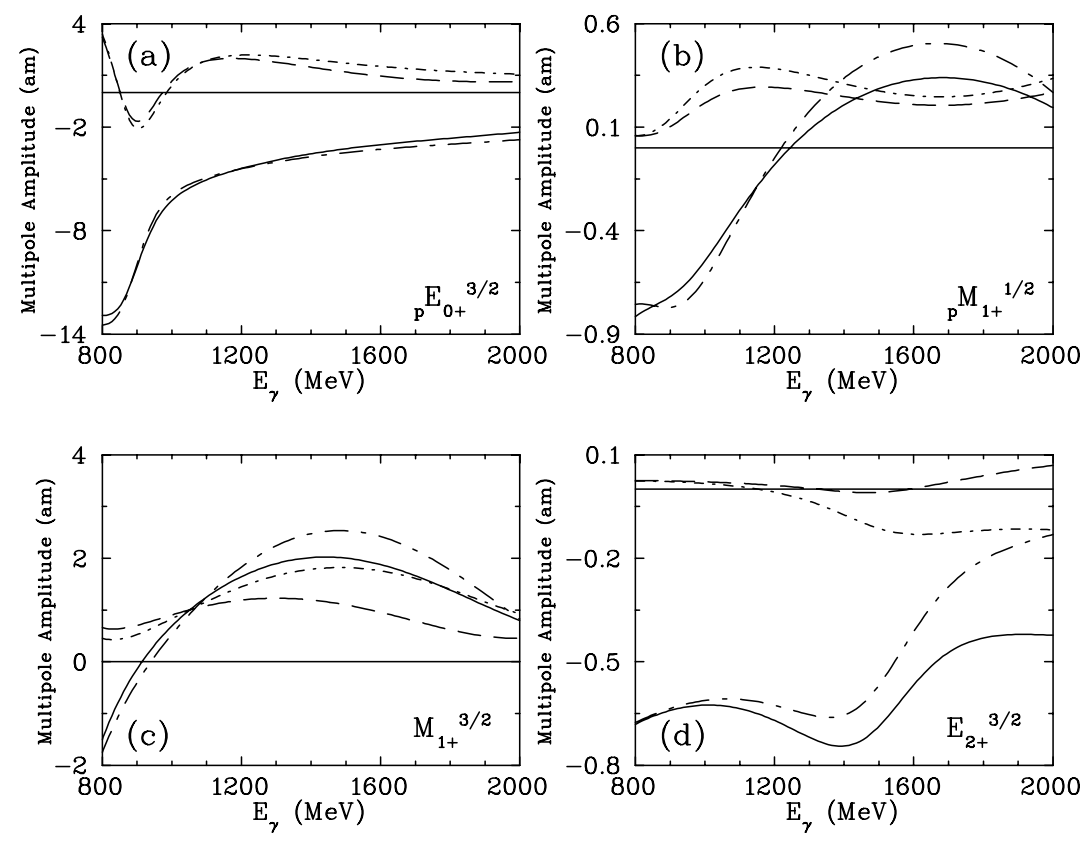

FIG. 5: Selected multipole amplitudes. Solid (dashed) curves give the real (imaginary) parts of amplitudes corresponding to the SM02 [1] solution. The real (imaginary) parts of the forced SC02 solution is plotted as dash-dotted (short dash-dotted) curves. 